\title{
Diarylheptanoids from the Roots of Juglans mandshurica
}

\author{
Gao Li, ${ }^{\dagger+}$ Chang-Seob Seo, ${ }^{\dagger}$ Seung-Ho Lee, ${ }^{\dagger}$ Yurngdong Jahng, Hyeun-Wook Chang, \\ Chong-Soon Lee, ${ }^{\$}$ Mi-Hec Woo, and Jong-Keun Son ${ }^{i t, *}$ \\ College of Pharmacy, Yeungnam University; Gyongsan 7/2-719, Korea \\ *College of Pharmacy, Yanbian Lniversity, Yanji 133000, P.R. China \\ Department of Biochemistr, College of Nantal Sciences, Yeungnam Liversity, Gyongsan 712-749. Korea \\ ${ }^{5}$ College of Pharmaci, Calholic University of Daegu, Gyongsan 712-702, Korea \\ Recened October 7. 2003
}

Key Words : Juglans mandshurica, Juglandaceae, Diarylheptanoid

The roots of Jiglans mandshurica Maximowicz (Juglandaceae) have been used as a folk medicine for the treatınent of cancer in Korea. Several naphthoquinones, naphthalenyl glucosides, tetralones, flavonoids, diarylheptanoid, and galloyl glycosides have been isolated from Juglans species. ${ }^{1 \cdot 10}$ These compounds have shown cytotoxic activity, topoisomerases I and II inhibitory activity, and inhibitory effect on both DNA polymerase and RNase $H$ activity of HIV-I reverse transcriptase. ${ }^{12-16}$ In the continuation of our studies on this plant, we isolated three new diarylheptanoids (1-3) from the $\mathrm{CHCl}_{3}$ fraction of the $\mathrm{MeOH}$ extract. This paper describes the structural determination of three new diarylheptanoids, and the absolute configurations of $\mathbf{1}$ and $\mathbf{3}$ were elucidated by Mosher's esters.

Three diarylheptanoids (1-3) were isolated from a $\mathrm{CHCl}_{3}$ fraction of the roots of $J$. mandshwica by repetitive column chromatography and preparative HPLC using a RP-18 column.

Compound 1 has the molecular formula $\mathrm{C}_{20} \mathrm{H}_{26} \mathrm{O}_{4}$ as determined by the HRFABMS, ${ }^{1.3} \mathrm{C}-\mathrm{NMR}$, and $\mathrm{DEP}$ '] spectral data. In the aromatic region of the ${ }^{1} \mathrm{H}-\mathrm{NMR}$ spectra of $1,{ }^{3}$ J coupling between $\mathrm{H}-5$ " and $\mathrm{H}-6$ ", and $3 /$ coupling between $\mathrm{H}-2^{\prime \prime}$ and $\mathrm{H}-6^{\prime \prime}$ indicated a 1,3,4-trisubstituted benzene ring, and ${ }^{2} J$ coupling between two sets of chemically equivalent protons $\left(\mathrm{H}-2{ }^{\prime} / \mathrm{H}-6^{\prime}\right.$ and $\left.\mathrm{H}-3^{\prime} / \mathrm{H}-5^{\prime}\right)$ suggested an I.4-disubstituted atomatic ring. The ${ }^{1:} \mathrm{C}$ NMR spectrum of 1 exhibited a total of 20 carbon signals, including characteristic signals due to a methoxyl group $\left(3 "-\mathrm{OCH}_{3}\right)$ and two chemically equivalent aromatic carbons ( $\mathrm{C}^{\prime}-2^{\prime} / \mathrm{C}^{\prime}-6^{\prime}$ and $\left(\mathrm{C}^{\prime}-3^{\prime} / \mathrm{C}^{\prime}-5^{\prime}\right)$. In the aliphatic region of DEPT spectra, one hydroxymethine and six methylene signals were exhibited. The ${ }^{\mathrm{i}} \mathrm{H}-{ }^{\mathrm{l}} \mathrm{H} \operatorname{COSY}$ spectrum showed connectivities among $\mathrm{H}-\mathrm{I}, \mathrm{H}-2, \mathrm{H}-3, \mathrm{H}-4$, $\mathrm{H}-5, \mathrm{H}-6$, and $\mathrm{H}-7$, between $\mathrm{H}-2^{\prime}\left(\mathrm{H}^{-} 6^{\prime}\right)$ and $\mathrm{H}-3^{\prime}\left(\mathrm{H}-5^{\prime}\right)$, and between $\mathrm{H}-5^{\prime \prime}$ and $\mathrm{H}-6$ ". In the HMBC spectrum of 1 (Figure 2), the connectivities of the two aromatic rings with the alkyl chain were indicated by the cross peaks between $\mathrm{H}-7$ and $\mathrm{C}-$ $\mathrm{I}^{\prime \prime}, \mathrm{C}-2^{\prime \prime}$ and $\mathrm{C}-6^{\prime \prime}$, and those between $\mathrm{H}-\mathrm{l}$ and $\mathrm{C}-\mathrm{I}, \mathrm{C}-\mathrm{C}^{\prime}$, and $C-6$ '. The position of the methoxyl group was determined by both the HMBC correlation of $\mathrm{C}-3^{\prime \prime}$ with 3 "-OCH and the positive NOE effect $(8.4 \%)$ between $\mathrm{H}-2$ " and $3 "$ -

\footnotetext{
"To whom correspondence should be addressed. Phone: +82-53-
} 810-2817; Fax:-82-53-8 ] l-387l; e-mail: jkson@yu.ac.kr<smiles>COc1cc([C]CCC[C@H](O)CCc2ccc(O)cc2)ccc1O</smiles>

1<smiles>COc1cc(/C=C/C=C/C(=O)CCc2ccc(O)cc2)ccc1O</smiles>

2<smiles>COc1cc(CC[C@@H](O)C[C@@H](O)CCc2ccc(O)cc2)ccc1O</smiles>

3

Figure 1. Diarylheptanoids isolated from the roots of Jtglans mantshurica.

$\mathrm{OCH}_{3} .^{17,18}$

The absolute stereochemistry of the chiral center in 1 was determined using the Mosher's ester based on the differences between the 'H-NMR chemical shifts of $(S)$ - and $(R)$-MTPA ester derivatives. 'H-NMR data were assigned based on the ${ }^{1} \mathrm{H}-{ }^{1} \mathrm{H}$ COSY spectra of $1_{\mathrm{s}}$ and $\mathbf{1}_{k}$ (Table 1). For 1, the negative value of $\Delta \delta_{1}\left(\delta-\delta_{R}\right)$ at $\mathrm{H}-2$ and the positive value of $\Delta \delta_{11}\left(\delta_{1}-\delta_{l l}\right)$ at $\mathrm{H}-4$ suggested a $R$ configuration at $\mathrm{C}-3$.

Compound 2 has the molecular formula $\mathrm{C}_{20} \mathrm{H}_{21} \mathrm{O}_{4}$ as determined by the HRFABMS, " ${ }^{1 .} \mathrm{C}-\mathrm{NMR}$, and DEP' spectral data. The 'H-NMR spectrum of 2 showed signals for a 1,3,4trisubstituted and a 1,4-disubstituted aromatic group same as compound 1. The ' $\mathrm{H}-{ }^{1} \mathrm{H}$ COSY spectrum of 2 showed the connectivities among $\mathrm{H}-4, \mathrm{H}-5, \mathrm{H}-6$, and $\mathrm{H}-7$, between $\mathrm{H}-1$ and $H-2$, between $H-2^{\prime}\left(H-6^{\prime}\right)$ and $H-3^{\prime}\left(H-5^{\prime}\right)$, and between $\mathrm{H}-5^{\mathrm{H}}$ and $\mathrm{H}-6^{\prime \prime}$. In the $\mathrm{HMBC}$ spectrum of 2 (Figure 2), the location of a carbonyl group in the chain was established by the correlations from $\mathrm{C}-3$ to $\mathrm{H}-2, \mathrm{H}-4$, and $\mathrm{H} 5$, and the connectivities of the two aromatic rings with the alkyl chain were indicated by the cross peaks from $\mathrm{H}-7$ to $\mathrm{C}-2^{\mu}$ and $\mathrm{C}-6^{\prime \prime}$ and from $\mathrm{H}-1$ to $\mathrm{C}-2^{\prime}$ and $\mathrm{C}-6$ ' The position of the methoxyl group on the aromatic ring was also determined by both the 
Table 1. Characteristic 'II-NMR data of MTPA esters of I and 3

\begin{tabular}{cccccccc}
\hline \multirow{2}{*}{ Position } & $\mathbf{1}_{s}$ & $\mathbf{1}_{k}$ & $\Delta \delta$ & Posi- & $\mathbf{3}_{k}$ & $\mathbf{3}_{k}$ & $\Delta \delta$ \\
& $\delta_{s}$ & $\delta_{k}$ & $\delta_{s}-\delta_{k}$ & tion & $\delta_{s}$ & $\delta_{k}$ & $\delta_{\mathrm{s}}-\delta_{\mathrm{k}}$ \\
\hline $\mathbf{1}$ & 2.51 & 2.72 & -0.09 & $\mathbf{1}$ & $\mathbf{2 . 5 7}$ & $\mathbf{2 . 6 2}$ & -0.05 \\
$\mathbf{2}$ & 1.91 & 2.01 & -0.10 & 2 & $\mathbf{2 . 0 0}$ & $\mathbf{2 . 0 7}$ & -0.07 \\
$\mathbf{3}$ & 5.13 & 5.17 & $\boldsymbol{R}$ & 3 & 5.46 & 5.32 & $\boldsymbol{S}$ \\
$\mathbf{4}$ & 1.79 & 1.71 & +0.08 & 4 & $\mathbf{2 . 1 8}$ & $\mathbf{2 . 0 6}$ & +0.12 \\
$\mathbf{5}$ & 1.45 & 1.32 & +0.13 & 5 & 5.46 & 5.32 & $\boldsymbol{S}$ \\
$\mathbf{6}$ & 1.68 & 1.59 & +0.09 & 6 & $\mathbf{1 . 9 7}$ & $\mathbf{2 . 0 0}$ & -0.03 \\
$\mathbf{7}$ & 2.65 & 2.55 & +0.10 & 7 & $\mathbf{2 . 5 7}$ & $\mathbf{2 . 6 2}$ & -0.05 \\
\hline
\end{tabular}

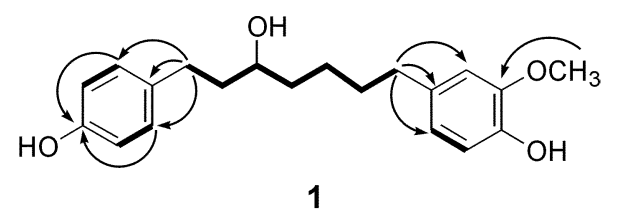

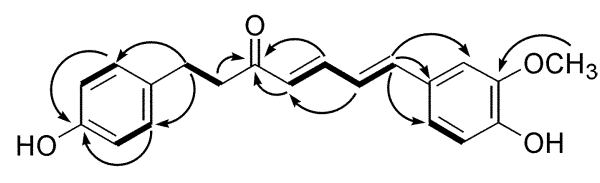

2

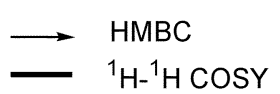

Figure 2, HMI3C and 'I-'II COSY correlations of compounds I and 2.

HMBC correlation between $\mathrm{C}-3$ " with $\mathrm{C}-3$ " $-\mathrm{OCH}_{3}$, and the positive NOE effect $(6.8 \%)$ between $\mathrm{H}-2^{\prime \prime}$ and $\mathrm{C}-3{ }^{\prime \prime}-\mathrm{OCH}_{3}$ in the ID-NOE difference spectrum of 2 .

${ }^{1} \mathrm{H}$ and ${ }^{13} \mathrm{C}$ NMR data of 3 was identical with those of reported compound which is an enantiomer of $3 .^{19}$ Io determine the absolute configuration of the hydroxyl groups at $\mathrm{C}-3$ and $\mathrm{C}-5$, MTPA esters ( $\mathbf{3}_{R}$ and $\mathbf{3}$ ) of $\mathbf{3}$ were prepared, and ${ }^{1} \mathrm{H}-\mathrm{NMR}$ data was also assigned based on the ${ }^{\prime} \mathrm{H}$.' $\mathrm{H}-$ COSY spectra (Table I). For 3 , the negative value of $\Delta \delta_{H}\left(\delta_{i-}\right.$ $\left.\delta_{k}\right)$ at $\mathrm{H}-\mathrm{I}$ and $\mathrm{H}-2$, and the negative value of $\Delta \delta_{\mathrm{H}}\left(\delta_{s}-\delta_{k}\right)$ at $\mathrm{H}-6$ and $\mathrm{H}-7$ suggested both $S$ configurations at $\mathrm{C}-3$ and $\mathrm{C}-5$.

Among these compounds, only 1 showed weak cytotoxicities against the HT-29 and MCF-7 cell lines (Table 2, $\mathrm{IC}_{50}$ : $>50 \mu \mathrm{g} / \mathrm{mL}$ and $47.7 \mu \mathrm{g} / \mathrm{mL}$, respectively).

Table 2, IC sil values of the compounds against H IT:-29 and MCl:-7 cell lines

\begin{tabular}{ccc}
\hline & \multicolumn{2}{c}{$\mathrm{IC}(\mu \mathrm{gg} / \mathrm{mL})$} \\
\cline { 2 - 3 } & $\mathrm{III}-2 y^{4}$ & $\mathrm{M}\left(\mathrm{F}-7^{h}\right.$ \\
\hline $\mathbf{2}$ & $>50$ & 47.7 \\
3 & $>50$ & $>50$ \\
$\mathrm{CPT}^{r}$ & $>50$ & $>50$ \\
& 0.035 & 3.5 \\
\hline
\end{tabular}

"IT-29: I fuman colon carcinoma. "MCF-7: H luman breast carcinumal camptolhesitr: positive control.

\section{Experimental Section}

General Experimental Procedures. Optical rotations were measured using a JASCO DIP-1000 (Tokyo, Japan) automatic digital polarimeter, and $\mathrm{F}]-[\mathrm{R}$ spectra were recorded on a JASCO F"T-IR 300E spectrophotometer. UV spectra were recorded on a JASCO V-550 spectrophotometer. For preparative HI'LC, LC-10AD pump (Shimadzu). SPD-10A detector (Shimadzu), and Shim-l’ack Prep-ODS $(20 \times 250$ $\mathrm{mm}$ ) column were used. NMR spectra were recorded on a Bruker $250 \mathrm{MHz}$ (DMX 250) spectrometer using Bruker's standard pulse program. Samples were dissolved in either acetone- $d_{6}$ or CD:OD, and chemical shifts were reported in ppın downfield from IMS. The MS spectra were measured by a VG TRIO 2A mass spectrometer. Silica gel $60(70-230$ and 270-400 mesh, Merck) and Lichroprep RP-18 gel (40$63 \mu \mathrm{m}$, Merck) were used for column chromatography. TLC plate (Silica-gel $60 \mathrm{~F}_{254}$ and $R P^{P}-18 \mathrm{~F}_{251}$ ) was purchased from EM Scientific. $(R)-(-)$ - $\alpha$-methoxy- $\alpha$-(trifluoromethyl) phenylacetyl [(R)-MTPA] chloride and (S)-(-)- $\alpha$-methoxy- $\alpha$ (trifluoromethyl)phenylacetyl [(S)-M[PA] chloride were purchased from Sigma Chemicals Co. Ltd. (St. Louis, MO, USA). All other chemicals and solvents were analytical grade, and used without further purification.

Plant Material. $J$. mondshurica roots were collected in September 1993 in a mountainous area of Pyongchanggoon, Gangwon-do, Korea, and dried at room temperature for 2 weeks. The material was confirmed taxonomically by Professor Gi-Hwan Bae, at Chungnam National University in Taejeon, Republic of Korea. A voucher specimen has been deposited at the College of l'harmacy, Yeungnam University.

Isolation. $J$. mandshurica roots ( $3 \mathrm{~kg}$ ) were extracted with $\mathrm{MeOH}$ two times under reflux for $12 \mathrm{~h}$ yielding $300 \mathrm{~g}$ of a dark solid extract. $280 \mathrm{~g}$ of which was then suspended in $\mathrm{H}_{2} \mathrm{O}$, and extracted with hexane. The resulting $\mathrm{H}_{2} \mathrm{O}$ layer was extracted with $\mathrm{CHCl}_{3}$, and the $\mathrm{CHCl}_{3}$ solution was evaporated to dryness in vacuo. The $\mathrm{CHCl}_{3}$ extract $(50 \mathrm{~g})$ was loaded on a silica gel column $(60 \times 9 \mathrm{~cm}$. Silica gel $70-$ 230 mesh), and the column was eluted with MeOH-EtOAc saturated with $\mathrm{H}_{2} \mathrm{O}$ (gradient from EtOAc $100 \%$ to $\mathrm{MeOH}$ $100 \%$ ). The eluent was combined on the basis of TLC. giving 17 fractions ( $\mathrm{Fl}-17)$. Fraction $\mathrm{F} 8(1.5 \mathrm{~g})$ was chromatographed on a reverse phase column $(60 \times 3.0 \mathrm{~cm}$. LiChroprep $\mathrm{RP}-18$ ) with $\mathrm{MeOH}-\mathrm{H}_{2} \mathrm{O}$ (gradient from $2: 8$ to $100 \%$ $\mathrm{MeOH}$ ), which afforded 22 subfractions (F8-1 8-22). Subfraction F8-3 $(250 \mathrm{mg})$ from the column was further purified on a reversed-phase column $(75 \times 2.0 \mathrm{~cm}$, LiChroprep RP18) with $\mathrm{MeOH}-\mathrm{H}_{2} \mathrm{O}$ (gradient from $10 \%$ to $90 \% \mathrm{MeOH}$ ). affording 1. Subfraction F8-6 (160 $\mathrm{mg})$ from the column was further purified on a reversed-phase column $(60 \times 2.0 \mathrm{~cm}$. LiChroprep RP-18) with $\mathrm{MeOH}-\mathrm{H}_{2} \mathrm{O}$ (gradient from $20 \%$ to $100 \% \mathrm{MeOH}$ ), affording compounds 2 and 3 . Further purifications of $\mathbf{1 - 3}$ were carried out using $\mathrm{HPLC}$ with $\mathrm{MeOH}-$ $\mathrm{H}_{2} \mathrm{O}$ gradients.

Compound 1: yellow solid $(15 \mathrm{mg}),[\alpha]_{\mathrm{b}}^{25}-12.3^{\circ}\left(c^{-}\right.$ $0.312 . \mathrm{MeOH}) ; \mathrm{UV}(\mathrm{MeOH}) \lambda_{\max }(\log \varepsilon) 223.2(4.13), 280.4$ (3.64), 347.0 (2.60); IR (KBr) v ทах 3391, 2933, 1613, 1514. 
1455, 1363. 1233. 1151, 1123. 1033. $825 \mathrm{~cm}^{-1}:{ }^{1} \mathrm{H}-\mathrm{NMR}$ (acetone $\left.-d_{6}, 250 \mathrm{MHz}\right) \delta 7.01\left(2 \mathrm{H} . \mathrm{d}, J=8.4 \mathrm{~Hz}, \mathrm{H}-2 / \mathrm{H}-6{ }^{\prime}\right)$. $6.77(\mathrm{lH} . \mathrm{d}, J=1.8 \mathrm{~Hz} . \mathrm{H}-2$ "). $6.72(2 \mathrm{H} . \mathrm{d}, J=8.4 \mathrm{~Hz}, \mathrm{H}-3$ ) $\left.\mathrm{H}-5^{\prime}\right) .6 .71\left(\mathrm{lH}, \mathrm{d} . J=8.0 \mathrm{~Hz}, \mathrm{H}-5^{\prime \prime}\right), 6.6 \mathrm{l}(\mathrm{lH}, \mathrm{dd} . J=8.0$. $\left.1.8 \mathrm{~Hz}, \mathrm{H}-6^{\prime \prime}\right), 3.79$ (3H. s, 3"- $\left.\mathrm{OCH}_{3}\right) .3 .31$ ( $1 \mathrm{H}$, br s, H-3). $2.61(2 \mathrm{H}, \mathrm{ml} . \mathrm{H}-1) .2 .50(2 \mathrm{H} . \mathrm{t}, J=7.5 \mathrm{~Hz} \mathrm{H}-7) .1 .68-1.43$ $(8 \mathrm{H}, \mathrm{m} . \mathrm{H}-2.6 .4 .5):{ }^{13} \mathrm{C}-\mathrm{NMR}$ (acetone- $\left.d_{6}, 62.9 \mathrm{MHz}\right) \delta$ 156.2 (C-4'). 148.1 (C-3"). 145.4 (C-4"), 134.9 (C-1'), 134.3 (C-1"). 130.1 (C-2/C-6'). 121.5 (C-6"). 115.9 (C-3/C-5'). $115.6\left(\mathrm{C}-5^{\prime \prime}\right) .112 .8\left(\mathrm{C}-2^{\prime \prime}\right) .70 .9(\mathrm{C}-3) .56 .2\left(3^{\prime \prime}-\mathrm{OCH}_{3}\right) .40 .8$ (C-2). 38.3 (C-4). 36.2 (C-7). 32.8 (C-6). 31.9 (C-1). 26.2 (C-5); HRFABMS $m=331.1911$. (calcd. for $\mathrm{C}_{3 \mathrm{i}} \mathrm{H}_{37} \mathrm{O}_{4}[\mathrm{M}+$ $\mathrm{H}^{-} .331 .1909$ ).

Compound 2: yellow solid $(1+\mathrm{mg})$; UV $(\mathrm{MeOH}) \lambda_{\text {max }}$ (log E) 272.4 (4.01). 361.2 (4.21); IR (KBI) v"max 3419. 2927. $1654,1610,1591,1514,1455,1384.1280 .1171 .1123$. $1031,827 \mathrm{~cm}^{-1}$ : ${ }^{1} \mathrm{H}-\mathrm{NMR}$ (acetone- $\left.d_{6} .250 \mathrm{MHz}\right) \delta 7.42$ $(1 \mathrm{H}$, dd. $J=15.4,9.4 \mathrm{~Hz}$. H-5). 7.21 (1H. d. $J=1.8 \mathrm{~Hz}$. H2"). $7.05\left(2 \mathrm{H}, \mathrm{d} . J=8.4 \mathrm{~Hz}, \mathrm{H}-2 / \mathrm{H}-6^{\prime}\right) .7 .0 \mathrm{l}(\mathrm{lH} . \mathrm{dd}, J=8.4$. $1.8 \mathrm{~Hz}$. H-6"). 6.98 (1H. dd. $J=15.4 .9 .4 \mathrm{~Hz}, \mathrm{H}-6$ ). 6.95 $(1 \mathrm{H}$, d. $J=15.4 \mathrm{~Hz} . \mathrm{H}-7), 6.81$ (lH. d. $\left.J=8.4 \mathrm{~Hz} . \mathrm{H}-5^{\prime \prime}\right)$. $6.72\left(2 \mathrm{H}, \mathrm{d}, J=8.4 \mathrm{~Hz} . \mathrm{H}-3^{\prime} / \mathrm{H}-5^{\prime}\right) .6 .23(\mathrm{lH}, \mathrm{d}, J=15.4 \mathrm{~Hz}$. $\mathrm{H}-4), 3.87\left(3 \mathrm{H} . \mathrm{s}, 3^{\prime \prime}-\mathrm{OCH}_{\mathrm{j}}\right), 2.84(2 \mathrm{H}, \mathrm{t}, J=5.3 \mathrm{~Hz}, \mathrm{H}-2)$. $2.81(2 \mathrm{H}$, t. $J=5.3 \mathrm{~Hz} . \mathrm{H}-1):{ }^{13} \mathrm{C}-\mathrm{NMR}$ (acetone- $d_{6 .} 62.9$ MHz) $\delta 199.3(\mathrm{C}-3) .156 .4\left(\mathrm{C}-4^{\prime}\right), 149.0\left(\mathrm{C}-3^{\prime \prime}\right), 148.7$ (C4 "), 143.8 (C-5). 142.3 (C-7). $133.0(\mathrm{C}-1 "), 130.0(\mathrm{C}-2 / \mathrm{C}-$ 6'). $129.3\left(\mathrm{C}-\mathrm{I}^{\prime}\right), 129.2(\mathrm{C}-4) .125 .2$ (C-6). 122.6 (C-6"). $116.0\left(\mathrm{C}-5^{\prime \prime}\right) .115 .9\left(\mathrm{C}-3^{\prime} / \mathrm{C}-5^{\prime}\right) .110 .5\left(\mathrm{C}-2^{\prime \prime}\right), 56.2\left(3^{\prime \prime} \cdot\right.$ $\left.\mathrm{OCH}_{3}\right) .42 .8(\mathrm{C}-2) .30 .1$ (C-1): HRFABMS $m z 325.1438$ (calcd. for $\mathrm{C}_{210} \mathrm{H}_{21} \mathrm{O}_{4}[\mathrm{M}+\mathrm{H}]^{-}, 325.1440$ ).

Compound 3: colorless amorphous solid (10 mg); $[\alpha]_{0}^{25}$ $+4.3^{\circ}(c=0.12, \mathrm{MeOH}) ; \mathrm{UV}(\mathrm{MeOH}) \lambda_{\max }(\log \varepsilon) 223.0$ (3.93). 280.0 (3.48); IR (KBr) $v_{\text {max }} 3340.2934,1613,1517$. $1454,1363.1233,1157.1032 .824 \mathrm{~cm}^{-1}:{ }^{1} \mathrm{H}-\mathrm{NMR}\left(\mathrm{CD}_{3} \mathrm{OD}\right.$. $250 \mathrm{MHz}) \delta 6.97\left(2 \mathrm{H}, \mathrm{d} . J=8.4 \mathrm{~Hz} . \mathrm{H}-2^{\prime} / \mathrm{H}-6^{\prime}\right), 6.74(1 \mathrm{H}, \mathrm{d}$. $\left.J=1.6 \mathrm{~Hz} . \mathrm{H}-2^{\prime \prime}\right), 6.66(1 \mathrm{H}, \mathrm{d} . J=8.0 \mathrm{~Hz}, \mathrm{H}-5 ") .6 .65(2 \mathrm{H}$. d. $\left.J=8.4 \mathrm{~Hz} . \mathrm{H}-3 / \mathrm{H}-5^{\prime}\right) .6 .59(\mathrm{lH} . \mathrm{dd}, J=8.0 .1 .6 \mathrm{~Hz}, \mathrm{H}-$ 6"), $3.79\left(3 \mathrm{H}, \mathrm{s} .3^{\prime \prime}-\mathrm{OCH}_{3}\right) .3 .79(2 \mathrm{H} . \mathrm{m}, \mathrm{H}-3.5), 2.70-2.46$ $(4 \mathrm{H}, \mathrm{m}, \mathrm{H}-1,7), 1.70-1.60$ (4H. m. H-2, 6). 1.51 (2H. t. $J=$ $6.1 \mathrm{~Hz}, \mathrm{H}-4):{ }^{13} \mathrm{C}-\mathrm{NMR}\left(\mathrm{CD}_{3} \mathrm{OD}, 62.9 \mathrm{MHz}\right) \delta 156.3\left(\mathrm{C}-4^{\prime}\right)$. 148.8 (C-3"). 145.7 (C-4"). 135.2 (C-1"). $134.4\left(\mathrm{C}-1^{\prime}\right) .130 .3$ $\left(\mathrm{C}-2^{\prime} / \mathrm{C}-6^{\prime}\right) .121 .8\left(\mathrm{C}-6^{\prime \prime}\right) .116 .1\left(\mathrm{C}-3^{3} / \mathrm{C}-5^{\prime}\right) .116 .0\left(\mathrm{C}-5^{\prime \prime}\right)$. $113.2\left(\mathrm{C}-2^{\prime \prime}\right) .68 .6(\mathrm{C}-3 / \mathrm{C}-5) .56 .3\left(3^{\prime \prime}-\mathrm{OCH}_{3}\right) .45 .6(\mathrm{C}-4)$. 41.4 (C-6). 41.3 (C-2) 32.6 (C-1). 32.1 (C-7): HRFABMS $m z 347.1814$ (calcd for $\mathrm{C}_{30} \mathrm{H}_{2 \jmath} \mathrm{O}_{4}\left[\mathrm{M}+\mathrm{H}^{-}, 347.1858\right.$ ).

Preparation of Mosher's Esters. To each $1 \mathrm{mg}$ of 1 and 3 in $0.5 \mathrm{~mL}$ of $\mathrm{CH}_{2} \mathrm{Cl}_{2}$ were added sequentially $0.2 \mathrm{~mL}$ of pyridine. $0.5 \mathrm{mg}$ of 4 -(dimethy lamino)pyridine. and $12.5 \mathrm{mg}$ of $(R)$-(-)- $\alpha$-methoxy- $\alpha$-(trifluoromethyl)pheny lacetyl $[(R)$ MTPA] chloride separately ${ }^{1022}$ The mixture was left at room temperature overnight and purified over a microcolumn $(0.6 \times 6 \mathrm{~cm})$ of silica gel $(230-400$ mesh) eluted with $3-4 \mathrm{~mL}$ of hexane- $\mathrm{CH}_{2} \mathrm{Cl}_{2}(1: 3)$. The elute was dried. $\mathrm{CH}_{2} \mathrm{Cl}_{2}(5$ $\mathrm{mL}$ ) was added and the $\mathrm{CH}_{2} \mathrm{Cl}_{2}$ was washed using $1 \%$
$\mathrm{NaHCO}_{3}(5 \mathrm{~mL} \times 2)$ and $\mathrm{H}_{2} \mathrm{O}(5 \mathrm{~mL} \times 2)$. The washed elute was dried in vacto to give the $S$-Mosher esters ( $\mathbf{1}_{S}$ and $\mathbf{3}_{\text {s) }}$ of $\mathbf{1}$ and $\mathbf{3}$, respectively. The $R$-Mosher esters $\left(\mathbf{1}_{R}\right.$ and $\left.\mathbf{3}_{k}\right)$ of $\mathbf{1}$ and 3 were prepared from $(S)$-MTPA chloride. respectively.

Cytotoxicity Bioassay. The tetrazolum-based colorimetric assay (MTT assay) was used for the in vitro assay of cytotoxicity against human colon carcinoma (HT-29) and human breast carcinoma (MCF-7) cells. ${ }^{-3}$

Acknowledgments. This work was supported by Korea Research Foundation Grant (KRF-2002-005-E00019).

\section{References}

1. Binder. R. G.: Benson. M. E.: Flash. R. A. Phytochemistry 1989. 28. 2799-2801.

2. Gupta. S. R.: Ravindranath, B: Seshadri, T. R. Phwochemistry 1972. $11,2634-2636$.

3. Muller. W.-U.: Leistner. E. Phytochemisty 1978, 17, 1739-1742.

4. Hirakawa. K:: Ogiue. E:: Motoyoshiya. J: Yajima. M. Phutochemisny 1986. 25. 1494-1495.

5. Talpatra. S. K.: Karmacharta. B.: De. S. C.: Talapatra. B Phtochemisny 1988, 27.3929-3932.

6. Pardhasaradhi. M.; Hari. B. M. Phtochemistry 1978, 17, 20422043.

7. Hedir1. P. A.: Collum. D. H.: Langhans. V. E.: Grave. C. H. $J$. Agric. Food Chem 1980. 28. 3401-342.

8. Son1. J. K. Awh. Pharn. Res. 1995. 18. 203-205.

9. Joe. Y. K; Son, J. K. Park, S. H.; Lee, I. J; Moon, D. C. J. Kat Prod 1996, 59, 159-160.

10. Lee. S. W.: Lee. K. S.; Son, J. K. Planta Med 2000, 66. $184-$ 186.

11. Li.G.: Xu. M. L.: Choi. H. G.: Lee. S. H.: Jahng. Y. D.: Lee. C. S.: Moon. D. C.: Woo. M. H.: Sont. T. K. Chent Pharn. Bull. 2003. 51. 262-264.

12. Kim. S. H.: Lee, K. S.: Son, J. K: Je, G. H. Lee, J. S.; Lee, C. H: Cheong. C. J. J. Wat. Prod. 1998. 61,643-645.

13. Lee. K. S.: Li. G.: Lee. C. S.: Woo. M. H.: Lee. S. H.: Jahng. Y. D.: Son1. T. K. J. Kat. Prod 2002. 65. 1707-1708.

14. Min. B. S.: Lee. H. K.: Lee. S. M.: Kim. Y. H.: Bae. K. H.: Otake. T.: Nakamura, N.; Hattori. M. Arch. Pham. Res. 2002. 25, 441445.

15. Min. B. S.: Nakamura, N.: Myashiro, H.: Kim. Y. H.; Hattoro. M. Chem. Pham Bull. 2000. 48. 194-200.

16. Li. G.: Lee. S. Y.: Lee. K. S.: Lee. S. W.: Kim. S. H.: Lee. S. H.: Lee. C. S.: Woo. M. H.: Son. J. K. Awh. Pham. Res. 2003. 26. 466-470.

17. Li. B. L.: Pan, Y. J. Bull. Korean Chem, Soc. 2002, 23. 617. 618.

18. Jeonl. Y. W: Jung. T. W: Kang. M: Chung. I. K.: Lee. W. Bull. Kowan Chem. Soc. 2002. 23.391-394.

19. Yokosuki. A.: Mimaki. Y.: Sakagami. H.: Sashida. Y. J. Nal. Prod. 2002. $65,283-289$.

20. Dale. J. A.: Mosher. H. S. J. Org Chem. 1973. 95. 512-519.

21. Rieser. M. J.; Hui. Y. H.: Rupprecht, J. K.: Kozlowshi. J. F.: Wood. K. V: McLaughlin. J. L.: Hansont. P. R.: Zhuang. A.: Hoye. T. R. J. Am. Chem. Soc. 1992. 14t. 10203-10213.

22. Ryu. G.: Choi. B. W.: Lee. B. H. Bull Korem Chem. Soc. 2002. 23. 1429-1434.

23. Rubinstein, L. V: Shoemaker. R. H.: Paul, K. D.: Simon. R. M.; Tosini. S.; Skehan, P: Scudiero, D. A.: Monks. A.; Boyd. M. R. $J$. Kat. Cancor Lnst 1990.82.1113-1118. 